\title{
Os Argulídeos (Crustacea: Branchiura) da Amazônia Brasileira, 2. Aspectos da ecologia de Dolops geayi Bouvier, 1897 e Argulus juparanaensis Castro, 1950. (1)
}

\author{
josé Celso de Oliveira Malta ${ }^{(2)}$
}

\begin{abstract}
Resumo
A especificidade parasitária, áreas de fixação, hospedeiros naturais, indices de infestação e variação sazonal de Dolops geayi e Argulus juparanaensis foram estudados durante o período de março de 1979 a março de 1980, na área do Janauacá, um lago de várzea da Amazônia Central. A sazonalidade revelou um estreito relacionamento com a variação do nível da água, sendo que o pico máximo de infestação do $\mathbf{D}$. geayi ocorreu na estação de rio cheio (junho) e o mínimo na estação de seca (outubro). O D. geayi apresentou uma certa especificidade parasitária ocorrendo em quatro espécies de peixes incluídas em três famílias, uma da Pimelodidae, uma da Erythrinidae e duas da Cichlidae. Devido a baixa ocorrência não foi possivel avaliar a especificidade e a sazonalidade de A. juparanaensis. Ambas espécies foram encontradas parasitando a cavidade branquial de seus hospedeiros.
\end{abstract}

\section{INTRODUÇÃo}

A subclasse Branchiura é formada por crustáceos ectoparasitas de peixes com aproximadamente 130 espécies conhecidas. O gênero Argulus é o que possui o maior número de representantes, cerca de 110, é cosmopolita e ocorre tanto em água doce quanto salgada. Os demais são dulcícolas e tem ocorrência restrita a determinadas regiões, tais como: Dolops, endêmico ao continente sul americano com onze espécies conhecidas, exceto, $D$. ranarum que só ocorre na África; o Chonopeltis é formado por sete espécies e tem ocorrência restrita ao continente africano; o Dipteropeltis é endêmico à América do Sul e possui apenas uma espécie; o Huargulus, também com apenas uma espécie, tem ocorrência restrita à China.
Os argulídeos são bastante conhecidos $\epsilon$ estudados em todo mundo, face aos danos que causam em populações naturais, confinadas e a peixes de aquários. Embora sejam inúmeras as dificuldades para se avaliar estes efeitos em condições naturais, certos autores conseguiram evidenciar alguns, tais como: redução do peso dos hospedeiros, da taxa de crescimento, da capacidade respiratória, da porcen tagem de músculos e gorduras e traumatização dos tecidos, facilitando o desenvolvimento de infecções secundárias (Kabata, 1970).

Em condições de cultivo intensivo de pei xes, os danos causados por crustáceos parasitas são aumentados, devido a uma grande concentração de indivíduos em pequenas áreas, o que favorece o ataque de parasitas, observando-se uma grande incidência de organismos sobre um único hospedeiro. A época de repro. dução destes crustáceos ocorre em duas ou mais estações durante o ano, sendo a produção de ovos relativamente grande, desde algumas dezenas até cerca de 400. Além disto, seu ciclo é direto completando-se em algumas semanas. Estas características fazem com que a população aumente progressivamente, a ponto destes crustáceos constituirem verdadeiras pragäs (Sarig \& Lahrav, 1959).

A primeira das espécies estudadas neste trabalho, Dolops geayi foi descrita por Bouvier em 1897, a locslidade tipo é a Venezuela e o hospedeiro tipo é desconhecido. A segunda o Argulus juparanaensis foi descrita por Castro em 1950, a localidade tipo é a Lagoa Juparaná situada no município de Linhares no Estado do Espirito Santo (região sudeste do Brasil), os hospedeiros tipos são Astyanax bimaculatus e Pachyurus squamipinis.

(1) - Parte da Tese de Mestrado apresentada ao curso de pós-graduação do Instituto Nacional de Pesquisas da Amazônia e Fundação Universidade do Amazonas.

(2) - Instituto Nacional de Pesquisas da Amazônia, Manaus. 


\section{MATERIAL E MÉTODOS}

A metodologia utilizada está de acordo com Malta (1981 e 1982).

\section{Resultados}

Durante o perícdo de março da 1979 a março de 1980 reaiizou-se excursões mensais ao lago Janauacá. Um total de 1355 peixes incluídos em oitenta espécies fcram examinados minuciosamente visando observar a ocorrência de crustáceos branquiuros. Neste trabalho são analizados os dados de duas espécies coletadas durante este período: o Dolops geayi Bouvier, 1897 (fig. 1 e 2) e o Argulus juparanaensis Castro, 1950 (fig. 3 e 4).

A primeira, o D. geayi, foi encontrada parasitando quatrc espécies de peixes, sendo que duas são assinaladas como novos hospedeiros para este crustáceo. A relação dos hospedeiros com seus respectivos índices de parasitismo são apresentados na Tabela 1 . O $D$. geayi ocorreu com maior intensidade parasitando peixes de escamas e dos quatro hospedeiros, apenas um é peixe de couro (sem escamas) .

Os peixes que ocorreram como hospedeideiros de $D$. geayi foram: Megalodoras sp, dezessete exemplares foram examinados. sendo que duas são assinaladas como novos hospedo $82,0 \%$ de incidência do parasita, uma intensidade de infestação variando de $0-6$ e uma média de treze indivíduos por peixe; Creni. cichla sp, sete exemplares foram examinados. sendo que dois estavam parasitados, apresentanáo $29,0 \%$ de incidência do parasita, uma intensidade de infestação variando de $0-6$ e uma média de seis indivíduos por peixe; Hoplias malabaricus quarenta e nove foram examinados sendo que sete estavam parasitados, apresentando $14,0 \%$ de incidência, uma intensidade de infestaçăo variando de $0-6$ e uma média de dois crustáceos por peixe; Astronotus ocellatus, quarenta e três foram examinados sendo que quatro estavam parasitados, apresentando $9,0 \%$ de incidência, uma intensidade de infestação variando de $0-1 \mathrm{e}$ com uma média de um parasita por peixe.

O Megalodoras sp e o $A$. ocellatus são citados pela primeira vez como hospedeiros de D. geayi.

Embora o D. geayi tenha apresentado uma preferência por peixes de escamas, o Megalc. doras sp que é um Siluriforme (sem escamas), foi o hospedeiro que apresentou os maiores índices de infestação, sendo considerado o principal hospedeiro desta espécie.

O D. geayi foi uma espécie bastante abundante, esteve presente em nove dos treze meses de coletas, sendo coligidos um total de 214 exemplares. Comparando seus índices de ocorrência com a variação do nível da água, verificou-se haver uma sazonalidade na infestação dos hospedeiros, onde cs maiores indi. ces coincidem com o mais elevado nível da água (estação cheia) e os menores com o mais baixo nível da água (estação seca). O pico máximo de infestação ocorreu no mês de junho (cheia) e o mínimo em outubro, o mês de mais baixo nível da água (fig. 5).

O D. geayi foi sempre coletado parasitan. do a cavidade branquial de seus hospedeiros.

TABELA 1 - Relação dos peixes que ocorreram como hospedeiros de Dolops geayi Bouvier, 1897 com seus indices de parasitismo; coletados no lago Janauacá durante o período de março de 1979 a março de 1980.

$\begin{array}{lcccc}\text { Hospedeiros } & \begin{array}{c}\mathrm{N} \cdot \text { de Peixes } \\ \text { Examinados/Parasitados }\end{array} & \% \text { de Incidência } & \begin{array}{c}\text { Intensidade de Infestação } \\ \text { Variação }\end{array} \\ \text { Megalodoras sp. } & 17 / 14 & 82,0 & 0-6 & 13,0 \\ \text { Crenicichla sp. } & 7 / 2 & 29,0 & 0-6 & 6,0 \\ \text { Hoplias malabaricus } & 49 / 7 & 14,0 & 0-6 & 2,0 \\ \text { Astronotus ocellatus } & 43 / 4 & 9,0 & 0-1 & 1,0\end{array}$




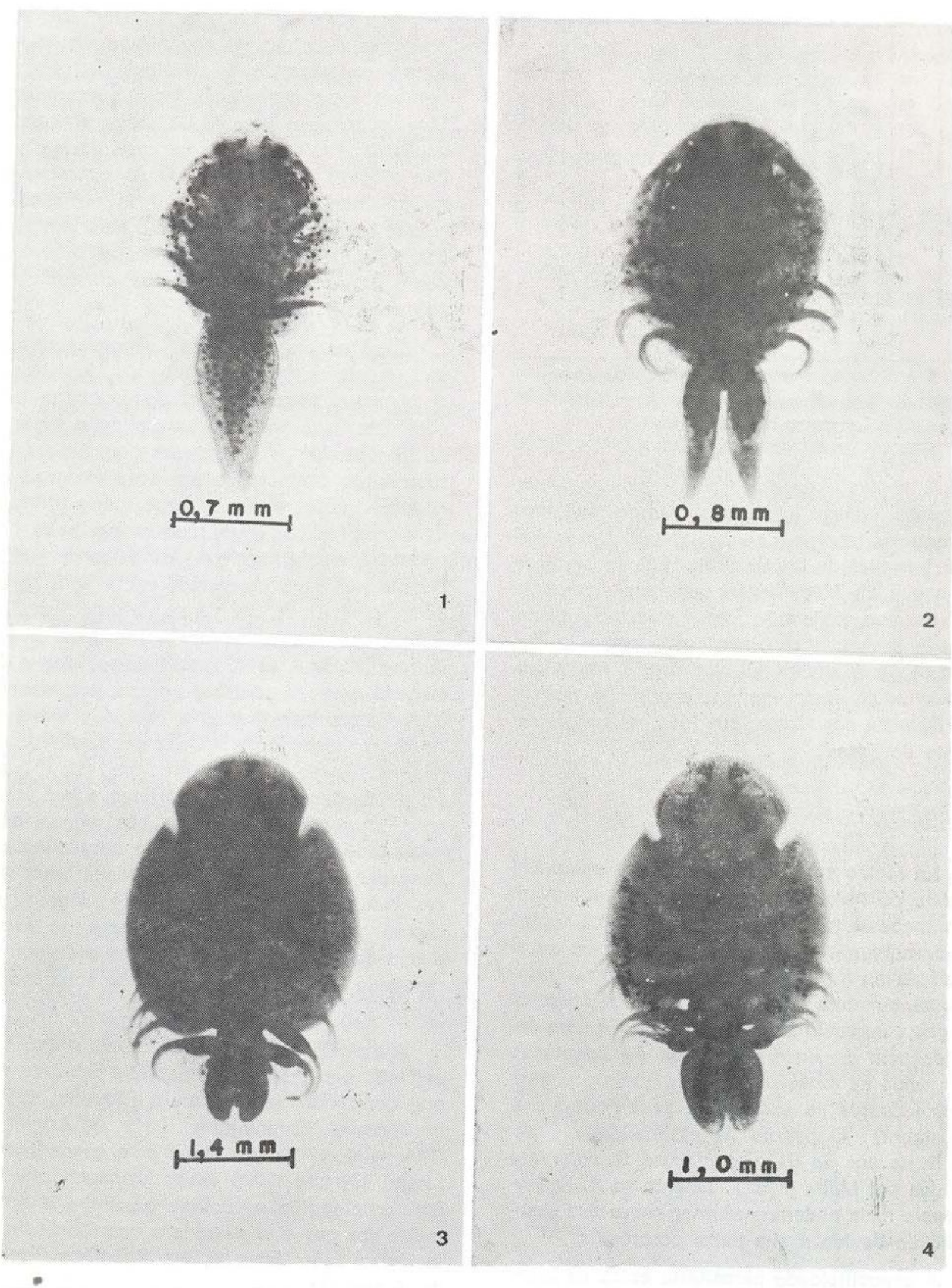

Fig. 1) Fêmea de Dolops geayi Bouvier, 1897; fig. 2) Macho de Dolops geayi Bouvier, 1897; fig. 3) Fêmea de Argulus juparanaensis Castro, 1950; fig. 4) Macho de Argulus juparanaensis Castro, 1950. 


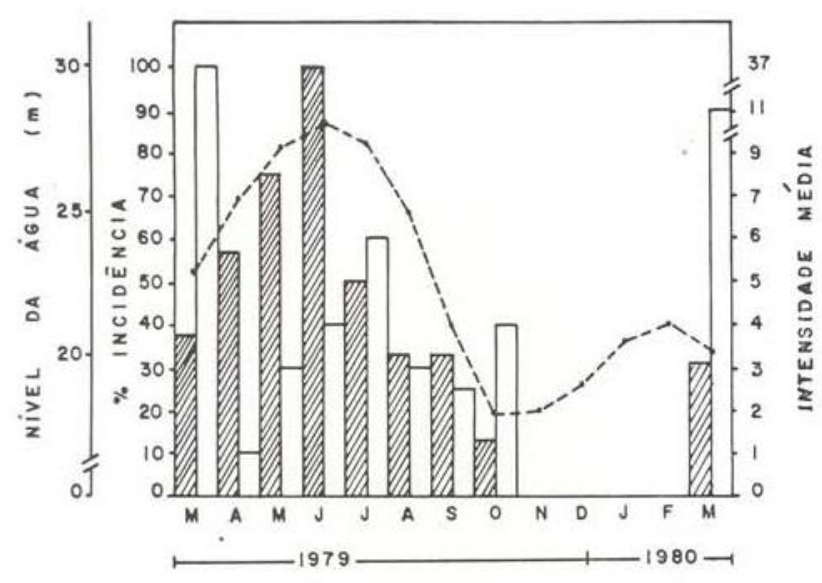

Fig. 5 - Variação sazonal de D. geayi relacionada com o nível da água. - incidência; $\square-$ intensidade; — nivel da água.

A segunda espécie, o $A$. juparanaensis, somente quatro exemplares foram coligidos durante os treze meses de coletas. Um exemplar foi coletado em abril de 1979 na cavidade branquial de Megalodoras sp, em agosto do mesmo ano, mais três foram coletados na ca. vidade branquial de Pseudoplatystoma fasciatum. Esta é a segunda vez que é registrada a ocorrência desta espécie, a primeira quando foi descrita por Castro em 1950, na região sudeste do Brasil.

\section{DISCUSSÃo}

Na região amazônica, as grandes variações anuais do nível da água dos rios, promovemi adaptaçōes à toda fauna e flora regional. Entre os branquiuros evidenciamos haver uma sazonalidade na infestação dos hospedeiros determinada por esta flutuação do nível da água. O $D$. geayi apresentou uma sazonalidade bem definida com os maiores índices de infestação ocorrendo na estação de cheia (maio e junho) e os menores na estação de seca (outubro e novembro). O padrão de sazonalidade é se melhante aos de $D$. discoidalis e $D$. bidentata citados por Malta (1982). Quarto ao A. jupara. naensis nada pedemos afirmar sobre sua sazonaliciade devido a sua baixa ocorrência.

A especificidade parasitária entre os branquiuros varia de espécie para espécie. Comparando o $D$. geayi com os crustáceos do lago Janauacá estudados por Malta (Ibid.), verifica- mos que este apresenta uma maior especifici dade que aqueles; foi encontrado parasitando quatro espécies de peixes incluídas em três famílias: uma espécie da familia Pimelodidae, Megalodoras sp; duas da Cichlidae, Astronotus ccellatus e Crenicichla sp e uma da Erythrinidae, Hoplias malabaricus. O A. juparanaensis foi encontrado parasitando duas espécies de peixes cada uma pertencendo a uma família de Siluriformes, mas devido a baixa ocorrência deste crustáceo nada podemos afirmar sobre sua especificidade.

Entre os branquiuros as segundas maxilas são transformadas em órgãos de fixação, sendo grossos apêndices arredondados na base com uma forte garra na extremidade superior, no gênero Dolops e ventosas com hastes quitinosas de sustentação em suas margens em Arguilus. Este grupo de crustáceos utiliza-se destes apêndices para fixarem em seus hospedeiros, embora entre as espécies do gênero Dolops não haja uma preferência para certas áreas específicas, ocorrendo de modo geral na superfície externa do corpo de seus hospedeiros (Malta, 1981), verificou-se que o $D$. geayı é exceção dentro do grupo, pois sempre foi coletado parasitando a cavidade branquial de seus hospedeiros. Os representantes do genêro Argulus por possuirem uma maior quantidacle de caracteres apomórficos e por terem apêndices mais eficientes, são encontrados parasitando áreas definidas do corpo de seus hospedeiros, tais como: a cavidade bucal e a cavidade branquial (Malta, 1981). Embora tenha-se coletado um baixo número de exemplares do A. juparanaensis, todos estavam parasitando a cavidade branquial de seus hospedeiros.

A distribuiçäo geográfica do $D$. geayi, res saltando a escassez de coletas, é bastante ampla, ocorrendo na Venezuela (Bouvier, 1897), no Paraguai (Cunnington, 1931), na Argentina (Ringuelet, 1943), no Brasil sem especificar a regiäo (Pearse, 1920 aplid: Yamaguti, 1963). Para uma região específica brasileira é a primeira vez que é registrada a ocorrência desta espécie: lago Januacá, rio Solimões, Estado do Amazonas.

A distribuição geográfica do A. juparanaensis evidencia a falta de estudos do grupo, sua 
localidade tipo é a cidade de Linhares no Estado do Espírito Santo, foi descrita em 1950 por Castro, só em 1980 foi novamente coletada. no lago Janauacá, Estado do Ảmazonas, sugerindo ocorrer em toda a região intermediária às duas localidades citadas.

Comparando as espécies de peixes que ocorreram como hospedeiras de $D$. geayi com aquelas que estavam parasitadas com o $D$. discoidalis e o $D$. bidentata (Malta, 1982): uma espécie é comum a todos os três crustá. ceos, o Astronotus ocellatus. Quanto aos hospederros do Argulus juparanaensis, uma espécie é comum ao $D$. discoidalis, o Pseudoplatystoma fasciatum e outra ao $D$. geayi, o Megalodoras sp.

\section{SUMMARY}

Host specificity, attachment sites, infection rates and seasonal variation of Dolpos geayi and Argulus juparanaensis were studied a one-year period, from March, 1979 , to March, 1980, in the vicinity of Janauacá, a várzea lake in Central Amazonia. Seasonal variation was found to be closely related to water levels, with the maximum infestation peak of D. geayi ocurring during high water (June) and minimum peak during low water (October). D. geayi showed a certain host specificity and was found in four fish species representing three families, namely: one specie of Pimelodidae, one of Erithrinidae and two of Cichlidae. It was not possible to evaluate the host specificity and seasonal variation of A. juparanaensis because the incidence of infestation was too low. Both species were found in the branchial cavities of their hosts.

\section{REFERẼNCIAS BIBLIOGRÁFICAS}

BOUVIER, M.E.L.

1897 - Observations sur les Arugulides du genre Gyropeltis recueillis par M. Geay au Venezuela. Bull. Mus. Hist. nat., Paris, 3 (1): 13-19.
CASTRO, A.L.

1950 - Contribuição ao estudo dos crustáceos argulídeos do Brasil. Descrição de duas novas espécies. Anais Acad. bras. Cien., 22 (2): $245-255$

CUNNINGTON, W.A.

1931 - Reports of expedition to Brasil and Paraguai in 1926-27. J. Linn. Soc., 37 (252): 259264.

KABATA, $Z$.

1970 - Diseases of fishes, Crustacea as enemies of fishes. Jersey City. TFH Publications, $171 \mathrm{p}$.

MALTA, J.C.O.

1981 - Os crustáceos branquiuros e suas interrelaçōes com os peixes do Lago Janauacá, Amazonas, Brasil (Crustacea, Argulidae). Tese de Mestrado. INPA-FUA. 88p.

1982 - Os argulídeos (Crustacea: Branchiura) da Amazônia brasileira. Aspectos da ecologia de Dolops discoidalis Bouvier, 1899 e Dolops bidentata Bouvier, 1899. Acta Amazonica. (no prelo).

RINGUELET, $R$.

1943 - Revision de los argulideos argentinos (Crustacea: Branchiura). Revta. Mus. la Plata, n. s., 3 (19): 43-125.

SARIG, G. \& LAHRAV, M.

1959 - The treatment with "Lindane" of carp and fish ponds infected with fish louse Augulus. Proc. tech. Pap. gen. Fisin Coun. Mediterr., 5: 151-156.

YAMAGUTI, S.

1963 - Parasitic Copepoda and Branchiura of fishes. New York. Interc. Public., 1103p.

(Aceito para publicação em 10/09/82) 